\title{
A Watermarking Method by Modifying QTCs for HEVC
}

\author{
Kwanghyok Jo ${ }^{a}$, Weimin $\mathrm{Lei}^{* \mathrm{~b}}$ and Zhaozheng $\mathrm{Li}^{\mathrm{c}}$ \\ College of Computer and Engineering, Northeastern University (NEU), Shenyang, China \\ ae-mail: zhaoguanghe2014@163.com; be-mail: leiweimin@ise.neu.edu.cn; \\ ce-mail:nooyung@163.com
}

Keywords: Index Terms-HEVC; QTCs; Watermarking

Abstract: With rapid development and application in the new video compression standard HEVC, it is necessary new digital watermarking technology capable of application for HEVC. In this paper, we propose a new watermarking method by modifying QTCs (Quantized Transform Coefficients) for HEVC. In proposed method, the watermark is embedded by modifying non-zero QTCs (Quantized Transform Coefficients) of the $4 \times 4$ luma transform block of intra predicted I-frame. In order to resist error drift phenomena, we differently modify the significant bits of $4 \times 4$ luma block with its direction of intra prediction modes. In decoder, the embedding watermark bits can extract without the need of any extra information. The experimental results show that proposed method is low in computational complexity and is efficiently resist error drift.

\section{Introduction}

With advances in digital video service and digital video processing technology, is the high risk associated with the ease of manipulation, tampering, redistribution and illegal copying of the digital content, so it is needed to prevent them. Watermarking technology plays an important role in securing multimedia data against illegal recording and retransmission [1].

High Efficiency Video Coding (HEVC) is the lastest video compression standard, developed by the Joint Collaborative Team on Video Coding (JCTVC), from ITU-T VCEG and ISO/IEC MPEG. One of main goals of HEVC is to provide approximately two times the compression efficiency in compared with H.264/AVC, while maintaining the same visual quality of H.264/AVC [2, 3]. Since HEVC has the probability of wide application, it is necessary for the research of the watermarking algorithms for it. However, HEVC is a video compression standard which it is recently proposed, so there are a little research works for its watermarking algorithms.

Video watermarking algorithm is closely related to video encoder structure. HEVC is built on H.264/AVC platform, therefore in general, it has been performed the research works for applying watermarking algorithms used in H.264/AVC to HEVC watermarking [7-20].

Since the HEVC encoder use some tools different from H.264/AVC, the watermarking algorithm using for H.264/AVC cannot directly apply to HEVC.

Among various watermarking algorithms, the algorithm that embed watermark to nonzero QTCs is highest in information embedding capacity and is robust for different attack [13-19]. In embedding watermark in QTCs, challenges are the error drift and the computational complexity. We try to 
propose the watermarking algorithm that computational complexity is low and resist error drift.

In this paper, considering the characteristic of intra prediction for HEVC, it is proposed a new digital watermarking algorithm. In proposed method, watermark is embedded by modifying QTCs of the $4 \times 4$ luma transform block of intra predicted I-frame. In order to resist error drift phenomena, we modify the significant bits of $4 \times 4$ luma block with its intra modes.

The rest of the paper is organized as follows. Section 2 briefly reviews the HEVC encoder and watermarking algorithms for HEVC. In section 3, the proposed algorithm is described, the experimental results and analysis are presented in section 4. Finally, conclusions are addressed in section 5 .

\section{Related Work}

\subsection{Introduction of HEVC}

HEVC is a successor to the H.264/AVC video coding standard [2,3]. HEVC adheres to the hybrid video coding structure; it uses spatial and temporal prediction, transform of the prediction residual, and entropy coding of the transform and prediction information. Figure 1 shows the structure of HEVC video encoder.

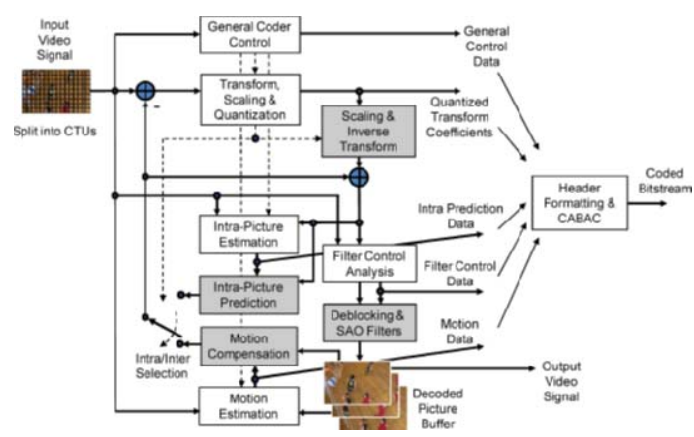

Fig. 1. The structure of HEVC video encoder

Fundamental difference between HEVC and previous video coding standards is that HEVC uses a quadtree structure [4]. The quadtree structure is a flexible mechanism for subdividing a picture into different block sizes for prediction and residual coding.

In HEVC, a block is defined as an array of samples. A unit encapsulates up to three blocks (e.g., one luma component block and its two corresponding chroma component blocks), and the associated syntactical information required to code these blocks. In HEVC, the basic processing unit is a coding tree unit (CTU). A CTU encapsulates up to three coding tree blocks (CTBs) and the related syntax. Each CTU has an associated quadtree structure that specifies how the CTU is subdivided. This subdivision yields coding units (CUs) that correspond to the leaves of the quadtree structure. A CU uses either intra or inter prediction and is subdivided into prediction units (PUs). For each PU, specific prediction parameters (i.e., intra prediction mode or motion data) are signaled. A nested quadtree, referred to as the residual quadtree (RQT), partitions a CU residual into transform units (TUs). The CUs, PUs, and TUs encapsulate coding blocks (CBs), prediction blocks (PBs), and transform blocks (TBs) respectively, as well as the associated syntax.

After the quadtree structure appropriately determines the TBs, a coded block flag signals whether a TB has any significant (i.e., nonzero) coefficient. If a TB contains significant coefficients, the residual coding process signals the position and value of each nonzero coefficient in the TB. A TB 
can range in size from $4 \times 4$ to $32 \times 32$ for luma and from $4 \times 4$ to $16 \times 16$ for chroma.

HEVC design supports a total of 35 intra prediction modes [5]. HEVC specifies two-dimensional transforms of various sizes from $4 \times 4$ to $32 \times 32$ that are finite precision approximations to the discrete cosine transform (DCT) [6]. In addition, HEVC also specifies an alternate $4 \times 4$ integer transform based on the discrete sine transform (DST) for use with 4x4 luma Intra prediction residual blocks.

A new tool in HEVC that improves coding efficiency is mode dependent coefficient scanning (MDCS) [6]. For intra coded blocks, the scanning order of a $4 \times 4$ TB and a $8 \times 8$ luma TB is determined by the intra prediction mode. In Table 1 , it is shown the mapping relation between Intra prediction modes and coefficient scanning order.

Table 1. Mapping Table Between Intra Prediction Modes and Coefficient Scanning Order

\begin{tabular}{|c|c|c|}
\hline Intra prediction mode & $\begin{array}{c}\text { Coefficient scanning for } \\
4 \times 4 \text { and } 8 \times 8\end{array}$ & $\begin{array}{c}\text { Coefficient scanning for } \\
16 \times 16 \text { and } 32 \times 32\end{array}$ \\
\hline Angular(6-14) & Vertical & Diagonal \\
Angular(22-30) & Horizontal & Diagonal \\
All other modes & Diagonal & Diagonal \\
\hline
\end{tabular}

Vertical and horizontal scans refer to the corresponding raster scan orders, while the diagonal scan refers to a diagonal scan from down-left to up-right direction.

\subsection{Related Work}

Similar to the H.264/AVC, the digital watermarking algorithms in HEVC can be divided into three main groups according to information embedding position: embedding in the stage of prediction, embedding in the stage of transform and quantification and embedding in the stage of entropy encoding.

(a) Embedding in the stage of prediction

In prediction stage, the elements that can modify to embed information are the intra prediction mode, the size of PU and the motion vector in inter prediction.

- Intra prediction mode

Wang et al. [7,8] proposed information embedding algorithms to embed information by modifying intra mode. It is modified the intra prediction modes in $4 \times 4$ luminance blocks to embed secret information [7]. According to the probability distribution of the statistical optimal prediction mode and suboptimal prediction mode, are establishing a mapping relationship between prediction modes and secret information, modified intra prediction modes to embed secret information based on the mapping. These schemes are complex and not efficient as the joint probability of optimal and sub-optimal mode is very low due to diversity of video texture.

It is established a mapping relationship table between the angle differences and secret information, based on this mapping, modified the intra prediction modes to embed secret information [8]. In this method, it is need to compute Lagrangian cost for all available prediction method, therefore computational complexity is relatively high and robustness is not considered.

- $\quad$ The size of PU

Compared to the H.264/AVC, HEVC gives up the concept of macroblock but introduces three basic uints: coding unit (CU), prediction unit (PU) and transform unit (TU). Tew et al. [9][10] accepted the information hiding method [11,12] using macroblock size in H.264/AVC to HEVC, and proposed an information hiding techniques using the coding block size decision in HEVC. This approach manipulates the $\mathrm{CB}$ (coding block) size decision on every coding tree unit to embed information based on the predefined mapping rules. Each $\mathrm{CB}$ is forced to assume certain size to encode the external information without significantly compromising perceptual quality. To improve 
payload, the odd-even based information hiding technique is further deployed by manipulating the nonzero DCT coefficients in certain ranges, in which case each range depends on the CB size. During encoding, the rate distortion optimizer (RDO) compute the cost function (i.e., a tradeoff between the distortion produced and the number of bits spent) of each possible block size for coding a given $\mathrm{PB}$ [9]. RDO selects the PB size with the lowest cost as the final decision to achieve the best compression ratio based on the desired bit rate. Instead of using the size suggested by RDO, the size of PB is forced to be the one representing the information to be embedded based on a predefined mapping rule. In particular, different PB size selection technique (i.e., PB Size) is applied when handling I, P and B-slices.

Although this technique prevents errors drift and the hiding capacity is high, the complexity is high due to the decoding and re-encoding step needed to embed the information into a pre-encoded video bit stream.

- Motion vector

Van et al. [15] suggested a low complexity out-of-the-loop information hiding algorithm for a video pre-encoded with the high efficiency video coding standard. Only selected components such as the motion vector difference and transform coefficients of the video are extracted and modified, bypassing the need of fully decoding and re-encoding the video. In order to reduce the propagation error caused by hiding information, the dependency between video frames is taken into account when distributing the information over the frame. The results show that embedding information in the transform coefficients only slightly outperforms the modification of motion vector differences.

(b) Embedding in the stage of transform and quantification

It is proposed information embedding algorithm that embed information to nonzero QTCs by a simple arithmetic operations [9, 13-15]. This method has the lack which is sensitive to the QP (quantization parameter) and don't consider the robustness.

Similar to H.264/AVC, when it changes the residual matrix for embedding of information, it exists the risk of generate error drift phenomenon because the HEVC encoder has the feedback loop. Therefore it should consider error drift when it changes the residual matrix.

Chang et al. [16] proposed the DCT/DST-based data hiding algorithm for HEVC intra-coded frames without propagating errors to neighboring blocks. This work is that extends Ma's work [17] for H.264/AVC for H.265/HEVC videos. Though, the scheme is drift compensated, it is fragile against re-compression attack.

It is presented the robust digital watermarking method for the HEVC based on Repetition BCH syndrome code technique without intra-frame distortion drift [18]. This method used the same algorithm as [15], the difference is to high the robustness of error channel by applying $\mathrm{BCH}$ encoding to watermark, but the embedding capacity is relatively low.

Dutta $T$ et al. [19] embedded a watermark bit in a $4 \times 4$ block in I frame by changing the value of first two nonzero AC coefficients.

It was proposed a blind watermarking scheme which embeds the watermark by altering the number of nonzero transform coefficients (NNZ) of $4 \times 4$ transform blocks of the HEVC video sequence [20]. However, the change of the number of NNZ may be impact the efficiency of entropy encoding.

(c) Embedding in the stage if entropy encoding

Bo Jiang et al. [21] proposed a CABAC based HEVC video steganography algorithm without bit rate increase. This algorithm performed information embedding through the codeword substitution during entropy coding. 


\section{Proposed Method}

The methods that embed the information in QTCs have been most widely used in information hiding algorithms because it is relatively large in embedding capacity and is robust. In embedding watermark in QTCs, challenges are the error drift and the computational complexity. We try to propose the watermarking algorithm that computational complexity is low and resist error drift.

The $4 \times 4$ luma block of intra predicted I frame is selected as watermarking embedding region in this paper. To embed watermark in intra predicted block is more robust than embedding of watermark in inter predicted block has been proven $[11,12]$. In addition, since I frames convey a wide range of information compared to $\mathrm{P}$ and $\mathrm{B}$ frames, it is important to protect them. The number of luma blocks is many than the number of chroma blocks, therefore it is favorable to extend embedding capacity.

In HEVC, there are four effective intra prediction block sizes ranging from $4 \times 4$ to $32 \times 32$ samples. Due to the characteristics of the human visual system, the video visual quality distortion caused by watermarking embedding is more visible in smooth regions while it is hard to detect in complex regions. It is used relatively large size of block for prediction in smooth regions. In contrary, it is used small size of block in complex regions. The size of block modify is more small, the impact of visual quality of video is more small. Therefore, We selected $4 \times 4$ luma block of intra predicted I frame as embedding region.

When it is modified QTCs, setting of scan order is related with the visual quality and the compression efficient of video. In H.264/AVC, the diagonal elements of QTC matrix are relatively more stable for modification than other elements. But in HEVC, the number of prediction modes is many than H.264/AVC, in particularly, the scanning order of a $4 \times 4 \mathrm{~TB}$ and a $8 \times 8$ luma TB is determined by the intra prediction mode. We refer the concept of MDCS defined in HEVC and differently selected the position of QTCs which have to change with their prediction mode. The position of watermarking embedding for different prediction mode in $4 \times 4$ block is shown in Fig. 2 .

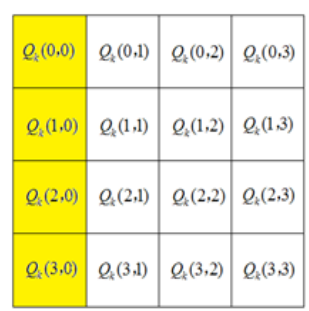

(a)

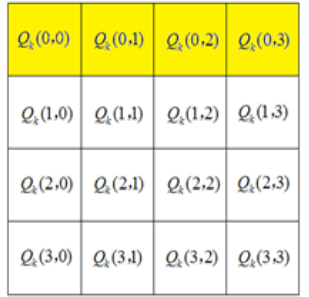

(b)

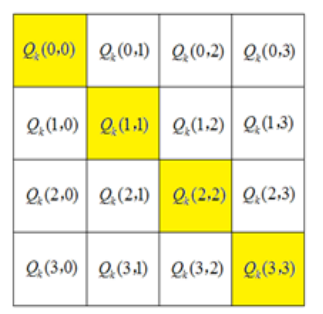

(c)

Fig. 2. Embedding position for different prediction mode ((a): case of intra prediction mode is 6 14; (b): case of intra prediction mode is 22 30; (c): else)

To prevent the negative effect which the change of QTCs gives entropy coding, we modify only coefficients which it is greater than 1 in significant bits of $4 \times 4$ luma blocks.

Embedding:

Step1: For intra predicted $4 \times 4$ luma block, it is selected the blocks where significant bits exist.

step2: Generate watermarking bits. The watermark information is binary random sequence denoted as $W=\left\{w_{k} \mid k=1,2, \ldots L, w_{k} \in[0,1]\right\}$.

Step3: Among selected blocks, for $Q_{k}(i, j)>1$, if intra prediction mode is $6 \sim 14$, then it is modified QTCs of vertical direction $Q(i, 0), i=0 \sim 3$. If Intra prediction mode is 22 30, then it is modified QTCs of vertical direction $Q(0, j), j=0 \sim 3$, else, it is modified QTCs of diagonal direction. 
Embedding formula is following:

For $Q_{k}(i, j)>1$

$$
\hat{Q}_{k}(i, j)=\left(Q_{k}(i, j)-Q_{k}(i, j) \bmod 2\right)+w_{k}
$$

Where $Q_{k}(i, j)$ and $\hat{Q}_{k}(i, j)$ is $i$ th row $j$ th column QTC of before embedding watermark and after embedding watermark in $k$ th block, respectively, and $w_{k}$ is $k$ th watermarking bit.

Extraction: For intra predicted $4 \times 4$ luma block, it firstly obtains the prediction mode information and selects the watermark extraction position based on the table1.

The extraction of watermarked bit is following;

For intra predicted $4 \times 4$ luma block, obtain information for prediction mode, select extraction position, then

if $\hat{Q}_{k}(i, j)>1$ then $w_{k}=\hat{Q}_{k}(i, j) \bmod 2$

\section{Experimental Results and Analysis}

The proposed algorithm has been simulated in the HM-16.9 model of the HEVC reference software. We select four different sequences (ParkScene_1920x1080.yuv, BasketballDrill_832x480.yuv, RaceHorses_416x240.yuv, FourPeople_1280x720.yuv) for testing. The main reference software coding configuration parameters are shown in Table 2 , and the remaining parameters are set to the default configuration.

Table 2. Configuration Parameters of the HM

\begin{tabular}{|c|c|}
\hline Parameters & Configuration \\
\hline Frames To Be Encoded & 30 \\
\hline Frame Rate & 96 \\
\hline Intra Period & 16 \\
\hline GOPSize & 8 \\
\hline RDOQTS & 1 \\
\hline
\end{tabular}

Fig. 3 shows the 16th I-frame of video before and after embedding of watermark. The original 16th I-frame is shown as Fig. 3(a), the 16th I-frame after embedding watermark is shown as Fig. 3(b). As shown in the picture, watermarking don't give any noticeable impact on perceptual video quality.

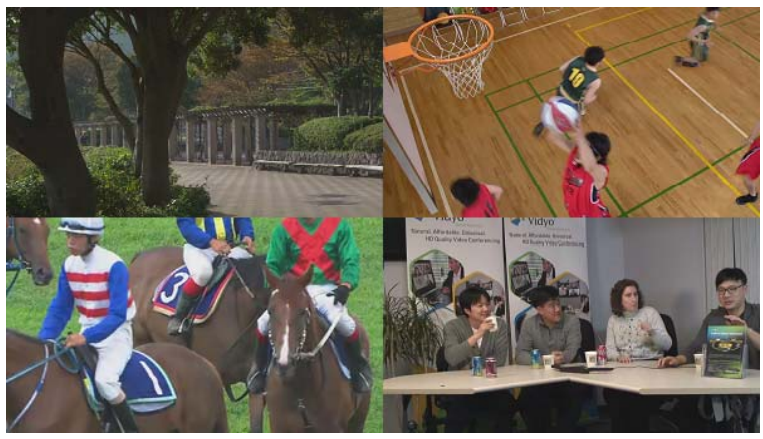

(a) 16th I-frame before embedding

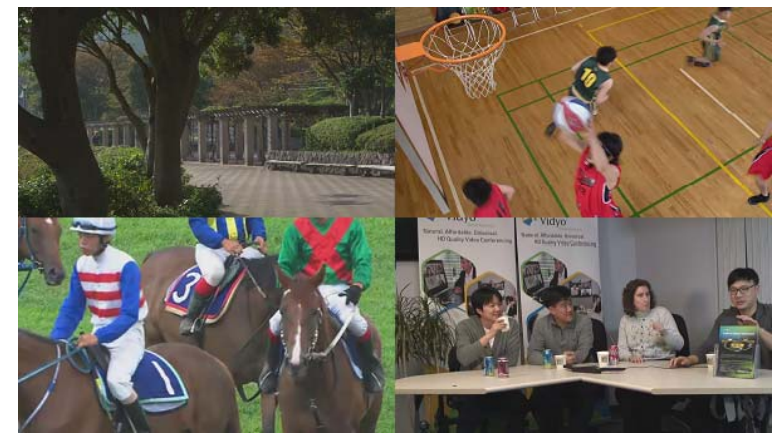

(b) 16th I-frame after embedding

Fig. 3. Comparison of the visual quality between the original and the watermarked frames. 
It is also calculated the objective video coding quality variation (PSNR) and bit rate variation (BIR) in experiments.

Peak Signal-to-Noise Ratio (PSNR) measure has been used to analyze the quality of watermarked video with respect to original video which is given by

$$
P S N R=10 \log _{10}\left(\frac{255^{2}}{M S E}\right)
$$

The difference of between the value of PSNR before embedding watermark and after embedding watermark $\triangle P S N R$ is defined by

$$
\triangle P S N R=P S N R_{Y}^{\prime}-P S N R_{Y}
$$

Where $P S N R_{Y}^{\prime}$ and $P S N R_{Y}$ are the video coding quality before and after embedded secret information respectively and MSE (mean square error) is a measure used to quantify the difference between the initial video frame $I$ and the distorted video frame $I^{\prime}$. If the video frame has a size of $\mathrm{M} \times \mathrm{N}$ then:

$$
M S E=\frac{1}{M N} \sum_{i=0}^{M-1} \sum_{j=0}^{N-1}\left(I(i . j)-I^{\prime}(i, j)\right)
$$

Table 3 shows the PSNR values of Y components $P S N R_{Y}$. As can be seen from the table, $P S N R_{Y}$ value is slightly lower after embedding the secret information, and the PSNR difference between original and embedded video is within $0.11 \mathrm{~dB}$. It is indicated that the proposed algorithm efficiently resist error drift.

Table 3. Comparison of $P S N R_{Y}$ value corresponding to different QP

\begin{tabular}{|c|c|c|c|c|c|c|c|c|c|}
\hline \multirow{2}{*}{ Sequence } & \multicolumn{3}{|c|}{$\mathrm{QP}=22$} & \multicolumn{3}{c|}{$\mathrm{QP}=32$} & \multicolumn{3}{c|}{$\mathrm{QP}=37$} \\
\cline { 2 - 10 } & $P S N R_{\mathrm{Y}}$ & $P S N R_{Y}^{\prime}$ & $\Delta P S N R$ & $P S N R_{Y}$ & $P S N R_{Y}^{\prime}$ & $\Delta P S N R$ & $P_{S N R_{Y}}$ & $P S N R_{Y}^{\prime}$ & $\Delta P S N R$ \\
\hline ParkScene & $\begin{array}{c}39.952 \\
1\end{array}$ & $\begin{array}{c}39.968 \\
3\end{array}$ & 0.0162 & $\begin{array}{c}34.852 \\
7\end{array}$ & $\begin{array}{c}34.918 \\
1\end{array}$ & 0.0654 & $\begin{array}{c}32.376 \\
8\end{array}$ & $\begin{array}{c}32.460 \\
4\end{array}$ & 0.0836 \\
\hline $\begin{array}{c}\text { BasketballDril } \\
1\end{array}$ & $\begin{array}{c}40.677 \\
9\end{array}$ & $\begin{array}{c}40.707 \\
9\end{array}$ & 0.0300 & $\begin{array}{c}34.682 \\
1\end{array}$ & $\begin{array}{c}34.754 \\
1\end{array}$ & 0.0720 & $\begin{array}{c}32.196 \\
4\end{array}$ & $\begin{array}{c}32.288 \\
6\end{array}$ & 0.0922 \\
\hline RaceHorses & $\begin{array}{c}39.168 \\
9\end{array}$ & $\begin{array}{c}39.189 \\
7\end{array}$ & 0.0208 & $\begin{array}{c}31.919 \\
6\end{array}$ & $\begin{array}{c}32.005 \\
6\end{array}$ & 0.0860 & $\begin{array}{c}29.128 \\
8\end{array}$ & $\begin{array}{c}29.238 \\
8\end{array}$ & 0.1100 \\
\hline FourPeople & $\begin{array}{c}42.708 \\
3\end{array}$ & $\begin{array}{c}42.718 \\
3\end{array}$ & 0.0100 & $\begin{array}{c}38.513 \\
3\end{array}$ & $\begin{array}{c}38.563 \\
3\end{array}$ & 0.0500 & $\begin{array}{c}35.746 \\
0\end{array}$ & $\begin{array}{c}35.833 \\
6\end{array}$ & 0.0876 \\
\hline
\end{tabular}

$B I R$ shows the percentage increase in bit rate that is defined as follows:

$$
B I R=\frac{R^{\prime}-R}{R} \times 100(\%)
$$

Where $R$ and $R^{\prime}$ is the bit rates generated by original encoder and modified encoder respectively.

Table 5 lists $B I R$ corresponding to different QP. On the average minimum bit rate increase is $0.126 \%$ in case of $\mathrm{QP}=22$ and maximum average increase is $0.758 \%$ in case of $\mathrm{QP}=37$.

Table 5. BIR corresponding to different QP ( $R$ for un-watermarked video, $R^{\prime}$ for watermarked video)

\begin{tabular}{|c|c|c|c|c|c|c|c|c|c|}
\hline \multirow{2}{*}{ Sequence } & \multicolumn{3}{|c|}{$\mathrm{QP}=22$} & \multicolumn{4}{c|}{$\mathrm{QP}=32$} & \multicolumn{3}{c|}{$\mathrm{QP}=37$} \\
\cline { 2 - 10 } & $R$ & $R^{\prime}$ & $B I R$ & $R$ & $R^{\prime}$ & $B I R$ & $R$ & $R^{\prime}$ & $B I R$ \\
\hline ParkScene & 11235.8250 & 11249.98 & 0.126 & 2324.8000 & $\begin{array}{c}2331.75 \\
1\end{array}$ & 0.299 & 1095.61 & 1100.869 & 0.480 \\
\hline $\begin{array}{c}\text { BasketballDril } \\
1\end{array}$ & 2312.6175 & $\begin{array}{c}2318.12 \\
2\end{array}$ & 0.238 & 562.0800 & $\begin{array}{c}564.024 \\
8\end{array}$ & 0.346 & $\begin{array}{c}299.342 \\
5\end{array}$ & 301.402 & 0.688 \\
\hline
\end{tabular}




\begin{tabular}{|c|c|c|c|c|c|c|c|c|c|}
\hline RaceHorses & 1395.7800 & 1399.66 & 0.278 & 345.8100 & $\begin{array}{c}347.148 \\
3\end{array}$ & 0.387 & $\begin{array}{c}172.645 \\
0\end{array}$ & $\begin{array}{c}173.953 \\
6\end{array}$ & 0.758 \\
\hline FourPeople & 1721.1875 & $\begin{array}{c}1723.45 \\
9\end{array}$ & 0.132 & 491.3475 & $\begin{array}{c}492.723 \\
3\end{array}$ & 0.280 & $\begin{array}{c}294.467 \\
5\end{array}$ & $\begin{array}{c}295.951 \\
6\end{array}$ & 0.504 \\
\hline
\end{tabular}

The PSNR difference and the bit rate difference between the original and embedded video is too small, that is, indicates that the algorithm induces limited quality degradation. It satisfies the visual concealment of the watermarking.

\section{Conclusions}

In this paper, we proposed a new robust watermarking algorithm. In proposed method, watermarking embed by modifying non-zero QTCs (Quantized Transform Coefficients) of the $4 \times 4$ luma transform block of intra predicted I-frame. In order to resist error drift phenomena, we differently modified the significant bits of $4 \times 4$ luma block in accordance with its direction of intra prediction modes. The embedded information bits can extract without the need of any extra information. The experimental results shows that the proposed method efficiently resists the error drift without extra computations.

\section{References}

[1] Ku, William, and C. H. Chi. "Survey on the Technological Aspects of Digital Rights Management." Lecture Notes in Computer Science 3225(2004):391-403.

[2] ITU-T Rec. "High efficiency video coding." H.265 and ISO/IEC 23008-2 (2013).

[3] Sullivan, G. J., et al. "Overview of the High Efficiency Video Coding (HEVC) Standard." IEEE Transactions on Circuits \& Systems for Video Technology 22.12(2012):1649-1668.

[4] Kim, Il Koo, et al. "Block Partitioning Structure in the HEVC Standard." IEEE Transactions on Circuits \& Systems for Video Technology 22.12(2012):1697-1706.

[5] Lainema, Jani, et al. "Intra Coding of the HEVC Standard." IEEE Transactions on Circuits \& Systems for Video Technology 22.12(2012):1792-1801.

[6] Sole, Joel, et al. "Transform Coefficient Coding in HEVC." Picture Coding Symposium IEEE, 2012:1765-1777.

[7] Wang, Jiaji, et al. "A Large-capacity Information Hiding Method for HEVC Video." International Conference on Computer Science and Service System 2014.

[8] Sheng, Qi, et al. "An Information Hiding Algorithm for HEVC Based on Differences of Intra Prediction Modes. Cloud Computing and Security." Springer International Publishing, 2015:1578-1585.

[9] Tew, Yiqi, K. S. Wong, and V. M. Baskaran. "Dual layer video stream in HEVC through information hiding." IEEE International Conference on Consumer Electronics - Taiwan 2015.

[10] Tew Y, Wong K S. "Information hiding in HEVC standard using adaptive coding block size decision." IEEE International Conference on Image Processing 2015.

[11] Yang, Gaobo, et al. "An information hiding algorithm based on intra-prediction modes and matrix coding for H.264/AVC video stream." AEU - International Journal of Electronics and Communications 65.4(2011):331-337.

[12] Hu, Yang, C. Zhang, and Y. Su. "Information Hiding Based on Intra Prediction Modes for H.264/AVC." IEEE International Conference on Multimedia and Expo IEEE, 2007:1231-1234.

[13] Mohamed, Mhamdi, et al. "Integration of a robust watermark scheme in a high efficiency codec H.265/HEVC with capacity-quality-bitrate trade-off." The International Conference 2015:375-379.

[14] Swati, Salahuddin, K. Hayat, and Z. Shahid. "A Watermarking Scheme for High Efficiency Video Coding 
(HEVC)." Plos One 9.9(2014):e105613-e105613.

[15] Van, Luong Pham, et al. "Out-of-the-loop information hiding for HEVC video." IEEE International Conference on Image Processing 2015:3610-3614.

[16] Chang, Po Chun, et al. "A DCT/DST-based error propagation-free data hiding algorithm for HEVC intra-coded frames 论." Journal of Visual Communication \& Image Representation 25.2(2014):239-253.

[17] Liu, Yunxia, et al. "A robust data hiding algorithm for H.264/AVC video streams." Journal of Systems \& Software 86.8(2013):2174-2183.

[18] Elrowayati, Ali A, et al. "Robust HEVC Video Watermarking Scheme Based on Repetition-BCH Syndrome Code." International Journal of Software Engineering \& Its Applications 10.1(2016):263-270.

[19] Dutta, Tanima, and H. P. Gupta. "A robust watermarking framework for High Efficiency Video Coding (HEVC) - Encoded video with blind extraction process 败." Journal of Visual Communication \& Image Representation 38(2016):29-44.

[20] Gaj, Sibaji, A. Sur, and P. K. Bora. "A robust watermarking scheme against re-compression attack for H.265/HEVC." Fifth National Conference on Computer Vision, Pattern Recognition, Image Processing and Graphics 2015.

[21] Jiang, B., G. Yang, and W. Chen. "A CABAC based HEVCc video steganography algorithm without bitrate increase." Journal of Computational Information Systems 11.6(2015):2121-2130.

[22] Information on https://hevc.hhi.fraunhofer.de/svn/svn_HEVCSoftware

[23] Flynn D, Rosewarne C. "Common test conditions and software reference configurations for HEVC range extensions." Proceedings of the 14th Meeting of Joint Collaborative Team on Video Coding (JCT-VC) of ITU-T SG16 WP3 and ISO/IEC JTC1/SC29/WG11 2013. 